\title{
Passeio aleatório unidimensional com ramificação em um meio aleatório K-periódico
}

Josué Macário de Figueiredo Rocha

DISSERTAÇÃO APRESENTADA

$\mathrm{AO}$

INSTITUTO DE MATEMÁTICA E ESTATÍSTICA

DA

UNIVERSIDADE DE SÃO PAULO

PARA

OBTENÇÃO DO GRAU DE MESTRE

EM

ESTATÍSTICA

Área de Concentração: PROBABILIDADE

Orientador: Prof. Dr. Fábio Prates Machado

São Paulo, 17 de Setembro de 2001

O autor recebeu auxílio financeiro da CNPQ. Processo 133164/1998-5 


\section{Dedicatória}

Ao meu avô, José de Figueiredo, pelos livros deixados na sala.

Ao meu pai, Macário Ivo de Brito Rocha, pelos livros, pelas palavras e pela cobrança.

À minha avó, Maria Nadege Fulco de Figueiredo, por ter transmitido continuamente e onde quer que estivesse que na vida é mais importante ter bons princípios do que quaisquer fins.

Ao meu irmão Virgílio Macário de Figueiredo Rocha $e$ às minhas mães, Célia Figueiredo, Leda Fulco de Figueiredo e Lúcia Fulco de Figueiredo, pela eterna torcida e infinita dedicação.

Aos Amigos que encontrei ou escolhi e aos bons Professores que, por sorte, tive. 


\section{Agradecimentos}

Agradeço a todos os meus familiares que são tão numerosos quanto maravilhosos e em especial às minhas mães.

"Tia Luceni", obrigado por não ter desejado o reconhecimento imediato da minha geração exigindo, além do que constava nos livros didáticos, que nos ensinassem valores familiares e princípios de cidadania. Madre Feitosa, obrigado por ser tão grandiosa e por me tratar como um príncipe.

Ao grande Prof. Robson Branco por me mostrar o poder da Matemática e como estudá-la de forma construtiva. Aos grandes professores Geovani e Rogério Porto por terem feito o mesmo com a Física.

Aos amigos que tenho em Recife (não ousarei enumerá-los). Aos amigos e aos bons professores que encontrei na Universidade Federal de Pernambuco.

Profa. Cristina, Prof. Cribari, Prof. Gauss e Prof. Klauss, obrigado por toda a receptividade, torcida e incentivo em meus primeiros estudos sobre estatística e em minha transição para a USP.

Agradeço ao Prof. Ednaldo Ernesto e ao "Dr. Luiz" por não admitirem o meu afastamento da Matemática e me redirecionarem não só com elogios, mas provando por $a+b$ que eu deveria estudá-la e praticá-la.

Aos amigos e aos bons professores que encontrei na USP e na cidade de São Paulo (não ousarei enumerá-los). Ao meu orientador, Prof. Fábio Prates Machado, pelo apoio, ensino e amizade. Ao prof. Serguei Yu. Popov pela receptividade e esclarecimentos fundamentais neste estudo. 
Gisela, obrigado por ser tão especial para mim.

Ao meu amigo Charles Casimiro Cavalcante por também admirar geometria e álgebra na época em que estudávamos para olimpíadas de Matemática e, desde então, sempre ter estado presente dispondo de sua atenção, amizade, inteligência e humildade para discutirmos idéias ou dividirmos alegrias. Meu eterno obrigado ao meu eterno Mestre, Prof. Ednaldo Ernesto, pela sua grandiosidade, por ser tão importante para a Matemática que existe em seus ex-alunos, por mostrar toda a poesia escondida na rainha das ciências e por transmitir a sua inexplicável humildade. Muito obrigado por vocês me suportarem.

Em fim, sou imensamente grato aos meus amigos. No fim, são só vocês que importam ... desde o começo. 


\section{Resumo}

Neste trabalho estudamos um passeio aleatório, unidimensional com ramificação em $\mathbb{Z}_{+}$em um meio aleatório não identicamente distribuído. Definimos recorrência e transiência para este processo e apresentamos um critério de classificação. 


\section{Abstract}

We study a "supercritical" branching random walk on $\mathbb{Z}_{+}$in a one-dimensional noni.i.d. random environment, which considers both the branching mechanism and the step transition. Criteria of (strong) recurrence and transience are presented for this model. 


\section{Índice}

Dedicatória $\quad$ ii

Agradecimentos iii

Resumo $\quad$ v

$\begin{array}{lll}\text { Abstract } & \text { vi }\end{array}$

1 Introdução 1

1.1 Descrição do Modelo . . . . . . . . . . . . . . . . . . . . . 2

1.2 Recorrência e Transiência neste Processo . . . . . . . . . . . . . 5

2 As Funções $h$ e $H \quad 8$

2.1 Definição . . . . . . . . . . . . . . . . . . . 8

2.2 Algumas Características . . . . . . . . . . . . . . 9

3 Recorrência x Transiência $\quad 18$

3.1 Principais Resultados . . . . . . . . . . . . . . . . . . . . . . 19

3.2 Um Lema Importante . . . . . . . . . . . . . . . . . . . . . . . . . . 20

3.3 A Condição Suficiente . . . . . . . . . . . . . . . . . . 22

3.4 A Condição Necessária . . . . . . . . . . . . . . . . . . . . . . . . . 24

Referências Bibliográficas $\quad 31$ 


\section{Capítulo 1}

\section{Introdução}

Estudamos aqui um processo markoviano a tempo discreto que consiste em passeios aleatórios com ramificação sobre $\mathbb{Z}_{+}$. Tanto os parâmetros dos passeios, quanto os parâmetros das ramificações são vetores aleatórios tomados de forma periódica a partir de um conjunto de $k$ distribuições de probabilidade sobre o espaço destes vetores.

Modelos similares são estudados em Bailon et al.[1], Menshikov et al.[11], Fleischmann e Greven[3], Greven e den Holander[5] e Révész[13]. Nos artigos Bailon et al.[1] e Greven e den Holander[5] são considerados sistemas com infinitas partículas e são obtidos resultados sobre a densidade global (local) de partículas. O limite de sistemas finitos com o número de incrementos de sítios são estudados em [3]. O principal objeto de estudo naquele trabalho é o tamanho da população total. Passeios aleatórios com ramificação em meio aleatório multi-dimensional são considerados em den Holander et al.[2] e Révész[13]. A densidade local de partículas é estudada em Révész[13], enquanto o foco principal de den Holander et al.[2] é recorrência e transiência.

Nos modelos descritos em Bailon et al.[1], Fleischmann e Greven[3], Greven e den Holander[5] e Révész[13] o meio aleatório leva em consideração apenas o mecanismo de ramificação. Passeio aleatório, unidimensional e com ramificação em meio aleatório identicamente distribuído e que leva em consideração tanto o mecanismo de ramificação como a transição entre sítios é visto em Menshikov et al.[11], onde também se tem uma classificação com respeito à recorrência e transiência. O presente trabalho consiste em generalizar os resultados de Menshikov et al.[11] para um meio aleatório não identicamente distribuído e se baseia em Machado e Popov [9].

Nossa principal ferramenta é o método de funções de Lyapunov que possibilita 
analisar o processo quanto à sua definição de transiência e recorrência de forma indireta, através do comportamento de funções definidas sobre o espaço onde o processo está definido. Embora não tratemos de outras aplicações do método de funções de Lyapunov neste trabalho, encontra-se na literatura exemplos de sua aplicação para o estudo de questões como taxa de convergência ao equilíbrio e estabilidade com relação aos parâmetros do processo. Veja por exemplo Fayolle et al. [4].

Em Comets, Menshikov e Popov[10] aplica-se este método para alguns processos markovianos sem ramificação em meio aleatório. Adaptações deste método para modelos com ramificação são encontrados em Menshikov et al.[11], Karpelevich et al.[6], Karpelevich e Suhov[7] e Menshikov e Volkov[12]; Também encontra-se em Karpelevich e Suhov[8] trabalho relacionado para o caso de espaço e tempo contínuos.

\subsection{Descrição do Modelo}

O processo aqui estudado se dá em tempo discreto e consiste no deslocamento de partículas que se ramificam nos sítios de $\mathbb{Z}_{+}=\{0,1,2, \ldots\}$ sem disputa de espaço e com as seguintes características:

(1) Estando em um sítio $x \neq 0$, num instante $t$, cada partícula gera pelo menos uma partícula filha e desaparece do processo.

(2) Cada partícula gerada desloca-se aleatoriamente para um dos sítios vizinhos mais próximos ou permanece em $x$ e, assim, chega-se a uma nova disposição de partículas no instante $t+1$ do processo.

(3) Cada partícula gera uma quantidade aleatória de filhas cuja distribuição de probabilidade $r$ depende somente da sua localização $x \in \mathbb{Z}_{+}$e onde:

(i) $r_{i}(x)$ é a probabilidade de uma partícula, estando no sítio $x$, gerar $i$ partículas filhas, para $i=1,2, \ldots$, isto é, temos:

$$
r_{i}(x):=P(\text { gerar } i \text { filhas } \mid \text { encontra-se na posição } x), \quad i=1,2, \ldots
$$


(ii) Naturalmente, por ser $r$ uma distribuição de probabilidade, temos para todo $x \in \mathbb{Z}_{+}$:

$$
r_{i}(x) \geq 0 \quad \text { e } \quad \sum_{i=1}^{\infty} r_{i}(x)=1
$$

(iii) $r(x):=\sum_{i=1}^{\infty} i r_{i}(x)<\infty$, para todo $x \neq 0$.

(4) Cada partícula filha gerada se encontrará no instante $t+1$ em um sítio $x_{t+1}$ de acordo com uma distribuição de probabilidade $P$, que só depende da localização $x_{t}$ da partícula mãe no instante $t$ e onde, para todo sítio $x \geq 1$ :

$$
\begin{aligned}
p_{x} & =P\left(x_{t+1}=x-1 \mid x_{t}=x\right) \\
s_{x} & =P\left(x_{t+1}=x \mid x_{t}=x\right) \\
& q_{x}=P\left(x_{t+1}=x+1 \mid x_{t}=x\right) \\
e \quad & p_{x}+s_{x}+q_{x}=1
\end{aligned}
$$

(5) O sítio zero possui as seguintes características:

$$
s_{0}=1 \quad \text { e } \quad r_{1}(0)=1
$$

(6) Os processos de geração de filhas e de transição entre os sítios são independentes entre as partículas.

(7) Seja M o conjunto de todas as seqüências $\xi=\left(p^{\xi}, s^{\xi}, q^{\xi}, r_{1}^{\xi}, r_{2}^{\xi}, r_{3}^{\xi}, \ldots\right)$ tais que

$$
p^{\xi}+s^{\xi}+q^{\xi}=1, \quad \sum_{i=1}^{\infty} r_{i}^{\xi}=1 \quad \text { e } \quad r^{\xi}:=\sum_{i=1}^{\infty} i r_{i}^{\xi}<\infty
$$

e $\tilde{Q}_{1}, \tilde{Q}_{2}, \ldots, \tilde{Q}_{k}, k$ distribuições de probabilidade sobre o conjunto M, satisfazendo:

- Condição I : $\quad \tilde{Q}_{i}\left(\xi \in M: p^{\xi} q^{\xi}=0\right)=0$, para todo $i$

- Condição II : $\quad \tilde{Q}_{i}\left(\xi \in M: r^{\xi}>1\right)>0$, para algum $i$.

Previamente ao início do processo, é associado a cada sítio $x \neq 0$ em $Z_{+}$um vetor $\xi_{x}=\left(p^{\xi_{x}}, s^{\xi_{x}}, q^{\xi_{x}}, r_{1}^{\xi_{x}}, r_{2}^{\xi_{x}}, r_{3}^{\xi_{x}}, \ldots\right)$, elemento do conjunto M, gerado pela distribuição $\tilde{Q}_{i}$ se $x$ é escrito da forma $n k+i \operatorname{com} n \in Z_{+}$e $1 \leq i \leq k$.

Associado $\xi_{x}$ ao sítio $x$, faz-se

$$
p_{x}=p^{\xi_{x}}, \quad q_{x}=q^{\xi_{x}}, \quad s_{x}=s^{\xi_{x}} \quad e \quad r_{i}(x)=r_{i}^{\xi_{x}}
$$


Seja uma seqüência de variáveis aleatórias $\boldsymbol{\xi}=\left\{\xi_{1}, \xi_{2}, \ldots\right\}, \xi_{i} \in M$ com as seguintes propriedades:

P1) Para qualquer $n \geq 0$ e $1 \leq i \leq k$ a variável aleatória $\xi_{n k+i}$ possui distribuição $\tilde{Q}_{i}$;

P2) $\left(\xi_{n k+1}, \xi_{n k+2}, \ldots, \xi_{n k+N}\right)$, com $N$ e $n \in Z_{+}$, é uma seqüência ergódica estacionária;

P3) Se $\mathbf{P}\left\{\xi_{x_{i}} \in A_{i}\right\}>0, i=1,2, \ldots, N$, então $\mathbf{P}\left\{\xi_{x_{1}} \in A_{1}, \ldots, \xi_{x_{N}} \in A_{N}\right\}>0$ para quaisquer $x_{1}, \ldots, x_{N} \in Z_{+}$e $A_{1}, \ldots, A_{N} \subset M$.

Esta seqüência chamamos de meio aleatório.

(8) Fixado o meio $\boldsymbol{\xi}:=\left\{\xi_{1}, \xi_{2}, \ldots\right\}$, o processo inicia-se com uma única partícula em um sítio $x_{o} \neq 0$.

Assim, pela propriedade $\mathrm{P} 1$, ao sítio 1 é associado um vetor $\xi_{1} \in M$ gerado pela distribuição $\tilde{Q}_{1}$, ao sítio 2 é associado um vetor $\xi_{2} \in M$ gerado pela distribuição $\tilde{Q}_{2}$, ... e ao sítio $k$ é associado um vetor $\xi_{k} \in M$ gerado pela distribuição $\tilde{Q}_{k}$. Ao sítio $k+1$ é associado um vetor $\xi_{k+1} \in M$ gerado, novamente, pela distribuição $\tilde{Q}_{1}$ e assim sucessivamente.

É neste sentido que o meio é periódico.

Desta forma, fixa-se o meio $\boldsymbol{\xi}=\left\{\xi_{1}, \xi_{2}, \ldots\right\}$ onde se desenvolverá o processo.

Com o meio $\boldsymbol{\xi}$ determinado, o processo inicia-se com uma única partícula em um sítio $x_{0} \neq 0$ e este representa o seu estado inicial. Esta partícula desaparecerá do processo após produzir no mínimo uma partícula filha segundo a distribuição $\tilde{r}$ determinada por $\xi_{x_{0}}$, vetor associado a $x_{0}$ pela distribuição $\tilde{Q}_{i}$ correspondente. Cada partícula desta primeira geração se deslocará de forma independente e sem disputa de espaço, para um dos sítios vizinhos, $x_{0}-1$ ou $x_{0}+1$, ou permanecerá em $x_{0}$ segundo as respectivas probabilidades $p_{x_{0}}, q_{x_{0}}$ e $s_{x_{0}}$, também determinadas por $\xi_{x_{0}}$.

Dispostas em $x_{0}-1, x_{0}$ e $x_{0}+1$, esta primeira geração, agora, caracterizará o estado do processo em $t=2$.

O espaço de estados deste processo é o conjunto

$$
\mathbb{Z}_{+} \cup \mathbb{Z}_{+}^{2} \cup \mathbb{Z}_{+}^{3} \cdots
$$


Um elemento deste conjunto, digamos

$$
\left(x_{t}^{1}, x_{t}^{2}, \ldots, x_{t}^{N(t)}\right)
$$

determina que no instante $t$ a $i$-ésima partícula se localiza na posição $x_{t}^{i}$, onde $N(t)$ é o número total de partículas neste instante.

Notemos que, para todo sítio $x \in \mathbb{Z}_{+}, r_{0}(x)=0$, pois

$$
\sum_{i=1}^{\infty} r_{i}(x)=1, \text { para todo } x \in \mathbb{Z}_{+},
$$

isto é, toda partícula é substituída por pelo menos uma partícula filha. Assim, $N(t)$ é não decrescente e $r^{\xi_{x}} \geq 1$ para todo $x \in \mathbb{Z}_{+}$.

Pela condição II, para algum $i, \tilde{Q}_{i}\left(\xi \in M: r^{\xi}>1\right)>0$. Como esta distribuição associa vetores aos sítios de $\mathbb{Z}_{+}$que pertencem ao conjunto $\{i, k+i, 2 k+i, \ldots\} \subset \mathbb{Z}_{+}$, por Borel-Cantelli, sabemos que, com probabilidade um, a uma quantidade infinita de sítios serão associados vetores $\xi_{x}$ tais que $r^{\xi_{x}}>1$. Assim, havendo sítio onde $r^{\xi_{x}}>1$, o processo mostra-se hábil em produzir ramificação.

Independente do estado inicial do processo, cada sítio de $\mathbb{Z}_{+}$possui probabilidade de ser visitado, após algum tempo. Isto é garantido pela condição I.

Se uma partícula desloca-se para o estado $\mathbf{0}$, devemos entender que a mesma é "congelada", não morrendo ou produzindo partículas descendentes no decorrer do processo. Mas, na verdade, $s_{\mathbf{0}}=1$ e $r_{1}(\mathbf{0})=1$.

\subsection{Recorrência e Transiência neste Processo}

Chamando de $N^{x}(t)$ o número de partículas que se encontram no sítio $x$ no instante $t$, vemos que $N^{\mathbf{0}}(t)$ é não decrescente, então o limite

$$
\nu=\lim _{t \rightarrow \infty} N^{0}(t)
$$

que pode assumir valor infinito, existe para toda realização do processo.

Claramente, $\nu$ é uma variável aleatória discreta não negativa.

Neste momento podemos definir recorrência e transiência para este processo. A mesma definição foi usada em Comets et al. [11]. 
Definição 1.2.1 Dizemos que o Processo é :

- Recorrente, se $P\{\nu \geq 1\}=1$;

- Fortemente Recorrente, se é recorrente e $E \nu<\infty$;

- Transiente, se $P\{\nu \geq 1\}<1$;

- Fortemente Transiente, se é transiente e $E \nu<\infty$.

A classificação do processo quanto à recorrência e transiência, segundo a definição acima, é independente do estado inicial.

Para vermos isto, suponhamos que existe um ponto inicial $x_{0}$ tal que, sendo esta a posição inicial do processo, seja positiva a probabilidade de termos $\nu=0$, isto é, $P_{x_{0}}\{\nu=0\}>0$ e portanto $P_{x_{0}}\{\nu \geq 1\}<1$.

Para qualquer outro ponto não nulo $x_{i} \in \mathbb{Z}_{+}$que fosse o sítio inicial do processo, a condição I garante ser positiva a probabilidade para que, após algum tempo, haja uma única partícula em $x_{0}$. Indiquemos esta probabilidade por $P_{x_{i}}^{x_{0}}$.

Mas

$$
P_{x_{i}}\{\nu=0\} \geq P_{x_{i}}^{x_{0}} P_{x_{0}}\{\nu=0\}>0 \Rightarrow P_{x_{i}}\{\nu=0\}>0,
$$

assim, se existe tal $x_{0}$ então $\nu$ tem probabilidade positiva de assumir valor nulo qualquer que seja o ponto inicial do processo.

Caso contrário, se existe um ponto $x_{0}$ tal que $P_{x_{0}}\{\nu=0\}=0$ e consequentemente $P_{x_{0}}\{\nu \geq 1\}=1$, isto será válido para qualquer outro ponto inicial $x_{i}$.

Assim, se para um determinado sítio $x_{0}$ temos $P_{x_{0}}\{\nu \geq 1\}<1$, então, independente do ponto inicial, $P\{\nu \geq 1\}<1$. Da mesma forma, se para um determinado sítio $x_{0}$ temos $P_{x_{0}}\{\nu \geq 1\}=1$, então $P\{\nu \geq 1\}=1$.

Suponhamos, agora, que existe $x_{0}$ tal que, se esta for a posição inicial do processo, seja infinita a esperança da variável $\nu$, isto é, $E_{x_{0}} \nu=\infty$.

Isto será válido para qualquer outro ponto inicial não nulo $x_{i}$ pois

$$
E_{x_{i}} \nu \geq P_{x_{i}}^{x_{0}} E_{x_{0}} \nu=\infty
$$


Caso contrário, se existe um ponto $x_{0}$ tal que $E_{x_{0}} \nu<\infty$, isto será válido para qualquer outro ponto inicial $x_{i}$.

Assim, se para um determinado sítio $x_{0}$ temos $E_{x_{0}} \nu=\infty$, então, independente do ponto inicial, $E \nu=\infty$. Da mesma forma, se para um determinado sítio $x_{0}$ temos $E_{x_{0}} \nu<\infty$, então $E \nu<\infty$.

Logo, a classificação do processo quanto à recorrência ou transiência é independente do seu estado inicial. 


\section{As Funções $h$ e $H$}

As funções definidas a seguir são de fundamental importância para a construção dos principais resultados deste estudo.

\subsection{Definição}

Definição 2.1.1 Para todo $\xi \in M$, seja

$$
h^{\xi}(\lambda)=\frac{1}{q^{\xi}}\left(\frac{1}{r^{\xi}}-s^{\xi}-\frac{p^{\xi}}{\lambda}\right), \quad \text { para } \quad 0<\lambda \leq \infty
$$

$e$, para $\lambda$ real, $i=1,2, \ldots, k$

$$
H_{i}(\lambda)=\left\{\begin{array}{cc}
\inf _{\xi \in \operatorname{supp} Q_{i}} h^{\xi}(\lambda) & \lambda>0 \\
-\infty & \lambda \leqslant 0
\end{array}\right.
$$




\section{$2.2 \quad$ Algumas Características}

Lema 2.2.1 $\quad$ (i) $\mathcal{D}_{i}:=\left\{\lambda \in \mathbb{R}: H_{i}(\lambda) \geq 0\right\}$ só pode assumir uma das duas formas:

$$
\begin{aligned}
& \mathcal{D}_{i}=\emptyset \\
& \mathcal{D}_{i}=\left[c_{i}, \infty\right) \text { para algum } c_{i}>0 .
\end{aligned}
$$

(ii) Existe $\mathbf{c}>0$ tal que para todo $\lambda<\mathbf{c}$ temos $H_{i}(\lambda)<0, i=1,2, \ldots, k$.

(iii) A função $H_{i}$ é contínua e não decrescente em $\mathcal{D}_{i}$ para $i=1,2, \ldots, k$.

Corolário 2.2.1 A função $\mathcal{H}:=H_{k} \circ \cdots \circ H_{1}$ é contínua e não decrescente em $\mathcal{D}:=\{\lambda: \mathcal{H}(\lambda) \geq 0\}$ que, como $\mathcal{D}_{i}$, só pode ser vazio ou da forma $\left[\lambda_{0}, \infty\right)$, para algum $\lambda_{0}>0$.

As demonstrações destas afirmações servem não só como esclarecimento, mas para nos ajudar a entender melhor o comportamento de $h$ e $H$, o que nos torna mais íntimos dessas funções.

\section{Prova do Lema 2.2.1}

(i) Por definição, se $\lambda \leq 0$ então $H_{i}(\lambda)=-\infty, \quad \forall i=1,2, \ldots, k$. Isto indica que, $\forall i=1,2, \ldots, k, \quad \mathcal{D}_{i} \subset(0, \infty)$

Teremos $\mathcal{D}_{i}=\emptyset$ quando, por exemplo, para qualquer $\lambda \in(0, \infty)$ exista um conjunto $A_{\lambda}:=\left\{\xi: h^{\xi}(\lambda)<0\right\}$ tal que $Q_{i}\left\{A_{\lambda}\right\}>0$. Desta forma, teremos $H_{i}(\lambda)<0$ para todo $\lambda>0$ e, conseqüentemente, $\mathcal{D}_{i}$ será vazio.

Exemplo:

Neste exemplo veremos que $\mathcal{D}_{i}$ será vazio porque $H_{i}(\lambda)$ é não só negativo para qualquer $\lambda \in(0, \infty)$, mas igual a $-\infty$. Suponha que para algum $i, Q_{i}$ seja a distribuição sobre o conjunto $\left\{\xi_{1}, \xi_{2}, \ldots, \xi_{n}, \ldots\right\}$ onde: 


$$
\begin{aligned}
p^{\xi_{n}} & =\frac{1}{2} \\
q^{\xi_{n}} & =\frac{1}{2^{n}} \\
s^{\xi_{n}} & =\frac{1}{2}-\frac{1}{2^{n}} \\
r^{\xi_{n}} & =\frac{n}{\frac{1}{2}+n s^{\xi_{n}}} \\
\text { e } \quad Q_{i}\left\{\xi_{n}\right\} & =2^{-n}, \quad n=1,2, \ldots
\end{aligned}
$$

Pela definição de $\xi_{n}$, vemos ser satisfeita a condição II para $Q_{i}$.

Mostremos que $r_{n}^{\xi}>1$ para todo inteiro positivo $n$.

Para todo $n \geq 1$, observa-se que

$$
\frac{n}{2} \geq \frac{1}{2} \quad \Rightarrow \quad n \geq \frac{n}{2}+\frac{1}{2} \quad \Rightarrow \quad n>\frac{n}{2}+\frac{1}{2}-\frac{n}{2^{n}}>0
$$

e portanto

$$
\frac{n}{\frac{n}{2}+\frac{1}{2}-\frac{n}{2^{n}}}>1
$$

mas

$$
\frac{n}{\frac{n}{2}+\frac{1}{2}-\frac{n}{2^{n}}}=\frac{n}{\frac{1}{2}+n\left(\frac{1}{2}-\frac{1}{2^{n}}\right)}=\frac{n}{\frac{1}{2}+n s^{\xi_{n}}}=r^{\xi_{n}}
$$

isto é, $r^{\xi_{n}}>1$.

Para esta distribuição temos que

$$
\begin{aligned}
& h^{\xi_{n}}(\lambda)=\frac{1}{q^{\xi_{n}}}\left(\frac{1}{r^{\xi_{n}}}-s^{\xi_{n}}-\frac{p^{\xi_{n}}}{\lambda}\right) \\
& h^{\xi_{n}}(\lambda)=2^{n}\left(\frac{\frac{1}{2}+n s^{\xi_{n}}}{n}-s^{\xi_{n}}-\frac{p^{\xi_{n}}}{\lambda}\right) \\
& h^{\xi_{n}}(\lambda)=2^{n}\left(\frac{1}{2 n}+s^{\xi_{n}}-s^{\xi_{n}}-\frac{p^{\xi_{n}}}{\lambda}\right) \\
& h^{\xi_{n}}(\lambda)=2^{n}\left(\frac{1}{2 n}-\frac{1}{2 \lambda}\right) \\
& h^{\xi_{n}}(\lambda)=2^{n-1}\left(\frac{1}{n}-\frac{1}{\lambda}\right)
\end{aligned}
$$


Vejamos que sob estas condições temos $\mathcal{D}_{i}=\emptyset$, como dissemos, sendo $H_{i}(\lambda)$ não só negativo para qualquer $\lambda \in(0, \infty)$, mas igual a $-\infty$.

Deve-se ter para quaisquer $\lambda \in(0, \infty)$ e $\rho \in(-\infty, 0)$ um conjunto $A_{\lambda}^{\rho}:=\{\xi$ : $\left.h^{\xi}(\lambda)<\rho\right\}$ tal que $Q_{i}\left\{A_{\lambda}^{\rho}\right\}>0$, pois assim $H_{i}(\lambda)=-\infty$ para todo $\lambda>0$ e, conseqüentemente, $\mathcal{D}_{i}$ será vazio.

Sabemos que para quaisquer $\lambda \in(0, \infty)$ e $\rho \in(-\infty, 0)$ existem

$$
n_{1}:=[\lambda+1] \quad \Rightarrow \quad\left(\frac{1}{n_{1}}-\frac{1}{\lambda}\right)<0
$$

e $n_{2}$ de tal modo que

$$
2^{n_{2}-1}\left(\frac{1}{n_{1}}-\frac{1}{\lambda}\right)<\rho
$$

Isto implica

$$
2^{N_{2}-1}\left(\frac{1}{N_{1}}-\frac{1}{\lambda}\right)<2^{n_{2}-1}\left(\frac{1}{n_{1}}-\frac{1}{\lambda}\right)<\rho, \quad \forall N_{1}>n_{1} \quad \text { e } \quad N_{2}>n_{2}
$$

Definindo $n_{0}=\max \left\{n_{1}, n_{2}\right\}$ vemos que

$$
h^{\xi_{n_{0}}}(\lambda)=2^{n_{0}-1}\left(\frac{1}{n_{0}}-\frac{1}{\lambda}\right)<\rho
$$

e como

$$
\rho>h^{\xi_{n_{0}}}(\lambda)>h^{\xi_{n_{0}+1}}(\lambda)>h^{\xi_{n_{0}+2}}(\lambda)>h^{\xi_{n_{0}+3}}(\lambda)>\cdots
$$

identificamos o conjunto $A_{\lambda}^{\rho}=\left\{\xi_{n_{0}}, \xi_{n_{0}+1}, \ldots\right\}$ onde

$$
\begin{aligned}
Q\left(A_{\lambda}^{\rho}\right) & =\sum_{n_{0}}^{\infty} Q\left(\xi_{n}\right) \\
& =\frac{1}{2^{n_{0}}}+\frac{1}{2^{n_{0}+1}}+\cdots \\
& =2^{1-n_{0}}>0
\end{aligned}
$$

Logo, para todo $\lambda \in(0, \infty), H_{i}(\lambda)=-\infty$ e, conseqüentemente, $\mathcal{D}_{i}=\emptyset$ 
Não sendo vazio, mostremos que $\mathcal{D}_{i}$ será um subconjunto próprio de $(0, \infty)$ irrestrito à direita, para $i=1, \ldots, k$ - isto é, $\mathcal{D}_{i} \subsetneq(0, \infty)$ e $\mathcal{D}_{i} \neq \emptyset$ - mostrando que existe $\lambda \in(0, \infty)$ e $\lambda \notin \mathcal{D}_{i}$.

Para $i=1, \ldots, k$, seja $\xi \in \operatorname{supp} Q_{i}$ onde $p^{\xi} \neq 0$ e $q^{\xi} \neq 0$.

Se $\lambda \in(0, \infty)$ tal que

$$
0<\lambda<\frac{p^{\xi}}{\left|\frac{1}{r^{\xi}}-s^{\xi}\right|}
$$

temos

$$
\begin{aligned}
& \lambda\left(\frac{1}{r^{\xi}}-s^{\xi}\right)<p^{\xi} \\
& \frac{1}{r^{\xi}}-s^{\xi}-\frac{p^{\xi}}{\lambda}<0 \\
& \frac{1}{q^{\xi}}\left(\frac{1}{r^{\xi}}-s^{\xi}-\frac{p^{\xi}}{\lambda}\right)<0,
\end{aligned}
$$

ou seja, $h^{\xi}(\lambda)<0$ e conseqüentemente $H_{i}(\lambda)<0$ e $\lambda \notin \mathcal{D}_{i}$. Logo $\mathcal{D}_{i} \subsetneq(0, \infty)$. Sendo $\mathcal{D}_{i} \neq \emptyset$, seja $\lambda_{1}$ um elemento qualquer deste conjunto e $\lambda_{2}>\lambda_{1}$. Assim

$$
\frac{1}{\lambda_{1}}>\frac{1}{\lambda_{2}} \Rightarrow-\frac{1}{\lambda_{1}}<-\frac{1}{\lambda_{2}}
$$

e, para qualquer $\xi=\left(p^{\xi}, s^{\xi}, q^{\xi}, r_{1}^{\xi}, r_{2}^{\xi}, \ldots\right)$, pertencendo ao suporte da distribuição $Q_{i}$, temos:

$$
-\frac{p^{\xi}}{\lambda_{1}} \leq-\frac{p^{\xi}}{\lambda_{2}} \Rightarrow \frac{1}{q^{\xi}}\left(\frac{1}{r^{\xi}}-s^{\xi}-\frac{p^{\xi}}{\lambda_{1}}\right) \leq \frac{1}{q^{\xi}}\left(\frac{1}{r^{\xi}}-s^{\xi}-\frac{p^{\xi}}{\lambda_{2}}\right)
$$

ou seja,

$$
h^{\xi}\left(\lambda_{1}\right) \leq h^{\xi}\left(\lambda_{2}\right) \quad \forall \xi \in \operatorname{supp} Q_{i}
$$

e portanto

$$
0 \leq H_{i}\left(\lambda_{1}\right) \leq H_{i}\left(\lambda_{2}\right) \quad i=1,2, \ldots, k
$$


Comprovamos assim que se $\lambda_{1} \in \mathcal{D}_{i}$

$$
\forall \lambda_{2}>\lambda_{1} \quad \text { temos } \quad \lambda_{2} \in \mathcal{D}_{i}, \quad i=1,2, \ldots, k
$$

ou seja, para todo $i$, se não é vazio, $\mathcal{D}_{i}$ é irrestrito à direita.

Embora seja subconjunto próprio de $(0, \infty)$ e irrestrito à direita, vejamos que não se pode ter $\mathcal{D}_{i}=\left(c_{i}, \infty\right)$ para algum $c_{i}>0$. Desta forma, não sendo vazio, $\mathcal{D}_{i}=\left[c_{i}, \infty\right) \subset(0, \infty)$ para $i=1,2, \ldots, k$.

Suponha que $\mathcal{D}_{i} \neq \emptyset$ e seja $\mathcal{D}_{i}^{\mathcal{C}}$ o complementar de $\mathcal{D}_{i}$ em relação ao conjunto dos números reais.

Para um elemento $\lambda \in \mathcal{D}_{i}^{\mathcal{C}}$ onde $\lambda>0$, mostremos que existe um número real $\epsilon>0$ tal que $\lambda+\epsilon \in \mathcal{D}_{i}^{\mathcal{C}}$.

Como

$$
\lambda \in \mathcal{D}_{i}^{\mathcal{C}} \Rightarrow H_{i}(\lambda)<0
$$

e como $\lambda>0$

$$
\exists \xi \in \operatorname{supp} Q_{i} \text { tal que } h^{\xi}(\lambda)<0 \text {. }
$$

Fixado $\xi$, a função $h^{\xi}$ é contínua em $(0, \infty)$ e, como visto em $(2.3)$, comporta-se não decrescente em $(0, \infty)$. Assim, existindo $\lambda$ positivo que torna $h^{\xi}(\lambda)<0$, existe $\epsilon>0$ tal que

$$
h^{\xi}(\lambda) \leq h^{\xi}(\lambda+\epsilon)<0 \text {. }
$$

Mas se $h^{\xi}(\lambda+\epsilon)<0$, então

$$
H_{i}(\lambda+\epsilon)<0 \Rightarrow \lambda+\epsilon \in \mathcal{D}_{i}^{\mathcal{C}} .
$$

Sendo $\mathcal{D}_{i}^{\mathcal{C}}$ aberto em $\mathbb{R}$, o seu complementar, o conjunto $\mathcal{D}_{i}$, é necessariamente da forma $\left[c_{i}, \infty\right)$.

Assim, para $i=1, \ldots, k$, se $\mathcal{D}_{i} \neq \emptyset$ então $\mathcal{D}_{i}$ será subconjunto de $(0, \infty)$ da forma $\left[c_{i}, \infty\right)$, para algum $c_{i}>0$. 
(ii) Como vimos no item (i), podemos ter $\mathcal{D}_{i}=\emptyset$ ou $\mathcal{D}_{i}=\left[c_{i}, \infty\right)$, para algum $c_{i}>0$.

Se $\mathcal{D}_{i}=\emptyset$, então $H_{i}(\lambda)<0$ para todo $\lambda \in(0, \infty)$ e portanto

$$
\forall c_{i} \in(0, \infty) \text { temos } H_{i}(\lambda)<0 \quad \text { se } \quad \lambda<c_{i} .
$$

Se $\mathcal{D}_{i}=\left[c_{i}, \infty\right)$, então $H_{i}(\lambda)<0 \quad$ se $\quad \lambda<c_{i}$.

Assim, vemos facilmente que definindo $\mathbf{c}=\underset{1 \leqslant i \leqslant k}{\operatorname{MIN}}\left\{c_{i}: \mathcal{D}_{i} \neq \emptyset\right\}$

$$
H_{i}(\lambda)<0 \quad \text { para todo } \quad \lambda<\mathbf{c} \quad \text { e } \quad i=1,2, \ldots, k,
$$

pois, neste caso, para $i=1, \ldots, k, \lambda \notin \mathcal{D}_{i}$ e portanto torna $H_{i}$ negativa.

(iii) Sobre o comportamento e continuidade da função $H_{i}$ em $\mathcal{D}_{i}$, pensemos na situação em que $\mathcal{D}_{i}=\left[c_{i}, \infty\right)$ para algum $c_{i}>0$, já que se $\mathcal{D}_{i}=\emptyset$ não temos nada a provar.

Para demonstrarmos o comportamento de $H_{i}$ em $\mathcal{D}_{i}$, sejam $\lambda_{1}<\lambda_{2}$ dois números reais não necessariamente pertencentes a $\mathcal{D}_{i}$.

Se $\lambda_{1} \leq 0$ temos $H_{i}\left(\lambda_{1}\right)=-\infty$ para $i=1,2,3, \ldots, k$ e portanto

$$
H_{i}\left(\lambda_{1}\right) \leq H_{i}\left(\lambda_{2}\right)
$$

Caso contrário, $0<\lambda_{1}<\lambda_{2}, \forall \xi \in \operatorname{supp} Q_{i}$ e $i=1,2,3, \ldots, k$, por (2.3), temos

$$
h^{\xi}\left(\lambda_{1}\right) \leq h^{\xi}\left(\lambda_{2}\right) \quad \Rightarrow \quad H_{i}\left(\lambda_{1}\right) \leq H_{i}\left(\lambda_{2}\right)
$$

Em síntese, para dois números reais $\lambda_{1}$ e $\lambda_{2}$

$$
\text { se } \quad \lambda_{1}<\lambda_{2} \Rightarrow H_{i}\left(\lambda_{1}\right) \leq H_{i}\left(\lambda_{2}\right) \quad i=1,2, \ldots, k \text {. }
$$

Logo, $H_{i}$ é não decrescente em $\mathbb{R}$ e portanto o é particularmente em $\mathcal{D}_{i}$ para $i=1,2, \ldots, k$.

Provemos a continuidade de $H_{i}$ em $\mathcal{D}_{i}$, para qualquer $i$.

Sabemos que, para um dado $\xi \in M, h^{\xi}$ é uma função contínua e não decrescente em $(0, \infty)$. Além disso, como

$$
\left(h^{\xi}(\lambda)\right)^{\prime}=\frac{p^{\xi}}{q^{\xi} \lambda^{2}}>0 \quad \forall \lambda \in(0, \infty),
$$


isto é, sendo sua derivada positiva e decrescente em $(0, \infty), h^{\xi}$ é côncava para todo $\xi \in M$. Desta forma, cada função $h^{\xi}$ determina um conjunto convexo, a região plana

$$
\mathcal{S}^{\xi}=\left\{(x, y) \in \mathbb{R}^{2}: x>0 \text { e } y \leq h^{\xi}(x)\right\},
$$

que é o conjunto dos pontos de $\mathbb{R}^{2}$ situados não acima do gráfico desta função. Qualquer que seja $i=1,2, \ldots, k$, notemos que

I) Para todo $x>0$, se $H_{i}(x)>-\infty$, então $\left(x, H_{i}(x)\right) \in \mathcal{S}^{\xi} \quad \forall \xi \in \operatorname{supp} Q_{i}$, pois $H_{i}(x) \leq h^{\xi}(x)$ para todo $\xi$ pertencente ao suporte da distribuição $Q_{i}$.

II) Se um elemento $\left(x, y_{1}\right) \in \mathcal{S}^{\xi}$ então, para todo $y_{2}<y_{1},\left(x, y_{2}\right) \in \mathcal{S}^{\xi}$, pois $y_{2}<y_{1}<h^{\xi}(x)$. Isto é, $\mathcal{S}^{\xi}$ não é limitada inferiormente.

Por I e II podemos afirmar que

$$
\left\{(x, y) \in \mathbb{R}^{2}: x>0 \text { e } y \leq H_{i}(x)\right\} \subset \mathcal{S}^{\xi} \quad \forall \xi \in \operatorname{supp} Q_{i},
$$

pois, qualquer que seja $x>0, H_{i}(x) \leq h^{\xi}(x) \quad \forall \xi \in \operatorname{supp} Q_{i}$.

Logo, definindo

$$
\mathcal{S}_{i}=\bigcap_{\xi \in \text { supp } Q_{i}} \mathcal{S}^{\xi}
$$

segue de 2.4 que

$$
\left\{(x, y) \in \mathbb{R}^{2}: x>0 \text { e } y \leq H_{i}(x)\right\} \subseteq \mathcal{S}_{i} .
$$

Além disso, notemos que

$$
\left\{(x, y) \in \mathbb{R}^{2}: x>0 \text { e } y>H_{i}(x)\right\} \cap \mathcal{S}_{i}=\emptyset,
$$

já que, $\operatorname{se}(x, y) \in \mathbb{R}^{2}, \operatorname{com} x>0$ e $y>H_{i}(x)$, existe $\xi^{*} \in \operatorname{supp} Q_{i}$ onde

$$
H_{i}(x) \leq h^{\xi^{*}}(x)<y
$$

e, portanto,

$$
(x, y) \notin \mathcal{S}^{\xi^{*}} \quad \Rightarrow \quad(x, y) \notin \mathcal{S}_{i} .
$$

Por 2.5 e 2.6, o gráfico de $H_{i}$ está contido na fronteira de $\mathcal{S}_{i}$ e limita superiormente esta região. $\mathcal{S}_{i}$ é um conjunto convexo por ser a intersecção de conjuntos 
convexos e constitui o conjunto dos pontos $(x, y) \in \mathbb{R}^{2}$, com $x>0$, situados não acima do gráfico da função $H_{i}$.

Assim, a função $H_{i}$ é contínua para todo $i$ em $\mathcal{D}_{i}$, pois, caso contrário, a região $\mathcal{S}$ não seria um conjunto convexo.

\section{Prova do Corolário 2.2.1}

A função $\mathcal{H}$ é claramente contínua e não decrescente em $\mathcal{D}$ por ser uma função composta de funções contínuas e não decrescentes.

Quanto a $\mathcal{D}$, notemos que para $\mathcal{H}(\lambda), \lambda \in(0, \infty)$, ser não negativa devemos ter

$$
\left(H_{i} \circ \cdots \circ H_{1}\right)(\lambda) \quad \in \quad \mathcal{D}_{i+1} \quad, \quad \forall i=1,2, \ldots, k-1
$$

já que, se $\left(H_{i} \circ \cdots \circ H_{1}\right)(\lambda) \notin \mathcal{D}_{i+1}$, para algum $i=1,2, \ldots, k-1$, teremos

$$
\left(H_{i+1} \circ H_{i} \circ \cdots \circ H_{1}\right)(\lambda)<0
$$

e portanto

$$
\begin{aligned}
& \left(H_{i+2} \circ H_{i+1} \circ H_{i} \circ \cdots \circ H_{1}\right)(\lambda)=-\infty \\
& \vdots \\
& \left(H_{k} \circ \cdots \circ H_{i+1} \circ H_{i} \circ \cdots \circ H_{1}\right)(\lambda)=-\infty,
\end{aligned}
$$

isto é,

$$
\mathcal{H}(\lambda)<0
$$

Naturalmente, temos que $\mathcal{D} \subseteq \mathcal{D}_{1}$, pois, como vimos acima, se $\lambda \in \mathcal{D}$ devemos ter $H_{1}(\lambda)>0$ (caso $i=1$ ) e portanto $\lambda \in \mathcal{D}_{1}$.

Pensemos inicialmente no caso em que $k=2$ para entendermos que $\mathcal{D}$ só pode ser vazio ou da forma $\left[\lambda_{0}, \infty\right)$, para algum $\lambda_{0}>0$.

Para $k=2, \mathcal{D}$ equivale ao conjunto $\left\{\lambda \in \mathcal{D}_{1}: H_{1}(\lambda) \in \mathcal{D}_{2}\right\}$. Analisemos dois casos:

1) $\mathcal{D}_{1}=\emptyset$ ou $\mathcal{D}_{2}=\emptyset$.

Se $\mathcal{D}_{1}=\emptyset$, então $\mathcal{D}=\emptyset$, já que $\mathcal{D} \subset \mathcal{D}_{1}$.

Se $\mathcal{D}_{2}=\emptyset$, então, para todo $\lambda,\left(H_{2} \circ H_{1}\right)(\lambda)<0$, pois $H_{1}(\lambda) \notin \mathcal{D}_{2}$.

Assim, neste caso, $\mathcal{D}=\emptyset$. 
2) $\mathcal{D}_{1}=\left[c_{1}, \infty\right) \subset(0, \infty)$ e $\mathcal{D}_{2}=\left[c_{2}, \infty\right) \subset(0, \infty)$.

2.1) Se $H_{1}(\lambda)<c_{2} \quad \forall \lambda \in \mathcal{D}_{1}$, então $H_{1}(\lambda) \notin \mathcal{D}_{2}$ e portanto $\left(H_{2} \circ H_{1}\right)(\lambda)<0$.

Assim, neste caso, $\mathcal{D}=\emptyset$.

2.2) Se $H_{1}(\lambda) \geq c_{2} \quad \forall \lambda \in \mathcal{D}_{1}$, ou seja, $H_{1}\left\{\mathcal{D}_{1}\right\} \subset \mathcal{D}_{2}$, então para todo $\lambda \in \mathcal{D}_{1},\left(H_{2} \circ H_{1}\right)(\lambda) \geq 0$.

Assim, neste caso, $\mathcal{D}=\mathcal{D}_{1}$.

2.3) Se $H_{1}\left(\lambda_{1}\right)<c_{2}<H_{1}\left(\lambda_{2}\right)$ para alguns valores $\lambda_{1}, \lambda_{2} \in \mathcal{D}_{1}$ supondo $c_{1} \leq$ $\lambda_{1}<\lambda_{2}$, então, pela continuidade e comportamento da função $H_{1}$, existe um elemento $\lambda_{0} \in \mathcal{D}_{1}$, onde $c_{1} \leq \lambda_{1}<\lambda_{0}<\lambda_{2}$, tal que $H_{1}\left(\lambda_{0}\right)=c_{2}$ e

$$
\begin{aligned}
& \forall \lambda<\lambda_{0} \quad \Rightarrow \quad H_{1}(\lambda)<c_{2} \\
& \forall \lambda>\lambda_{0} \quad \Rightarrow \quad H_{1}(\lambda) \geq c_{2}
\end{aligned}
$$

Isto é, existe um elemento mínimo, $\lambda_{0}$, em $\mathcal{D}_{1}$ de tal forma que $H_{1}\left(\lambda_{0}\right)=c_{2}$. Assim, neste caso, $\mathcal{D}=\left[\lambda_{0}, \infty\right)$.

De 1 e 2 obtemos que $\mathcal{D}=\emptyset$ ou $\mathcal{D}=\left[\lambda_{0}, \infty\right)$ para algum $\lambda_{0} \geq c_{1}$.

Suponha que isto seja válido para $k-1$, isto é, o subconjunto de $\mathcal{D} \subset \mathcal{D}_{1}$ tal que, se $\lambda \in \mathcal{D},\left(H_{k-1} \circ \cdots \circ H_{1}\right)(\lambda) \geq 0$ é vazio ou da forma $\left[\lambda_{0}, \infty\right)$ para algum $\lambda_{0} \geq c_{1}$.

Como sabemos, a função $H_{k-1} \circ \cdots \circ H_{1}$ é contínua e não decrescente; características também observadas na função $H_{1}$.

Como supomos, para a função $H_{k-1} \circ \cdots \circ H_{1}$, não há valores $\lambda$ tais que $\left(H_{k-1} \circ\right.$ .. $\left.\circ H_{1}\right)(\lambda) \geq 0$ ou este conjunto de valores é da forma $\left[\lambda_{0}, \infty\right) \subset[0, \infty)$. Da mesma forma, para a função $H_{1}, D_{1}$ é vazio ou igual a $\left[c_{1}, \infty\right) \subset[0, \infty)$.

Finalmente, para a função $H_{k}, D_{k}$ é vazio ou da forma $\left[c_{k}, \infty\right) \subset[0, \infty)$; similarmente, para a função $H_{2}, D_{2}$ é vazio ou igual a $\left[c_{2}, \infty\right) \subset[0, \infty)$.

Usando raciocínio análogo ao utilizado em 1 e 2 acima, identificando a função $H_{k-1} \circ \cdots \circ H_{1}$ com $H_{1}$ e a função $H_{k}$ com $H_{2}$, segue por indução que, para todo $k$ inteiro positivo, $\mathcal{D}=\{\lambda: \mathcal{H}(\lambda) \geq 0\}$ é vazio ou da forma $\left[\lambda_{0}, \infty\right)$ para $\operatorname{algum} \lambda_{0}>0$. 


\section{Recorrência x Transiência}

Segundo a nossa definição, um dado processo terá comportamento recorrente ou transiente (forte ou não) dependendo somente do meio $\xi$ onde o mesmo se desenvolve, já que, como vimos, a sua classificação não sofre influência da posição inicial da primeira partícula.

A nossa classificação diz respeito à probabilidade de partículas serem captadas no sítio 0 e à média do número de partículas que se encontram neste sítio após decorrido um tempo infinito - "a intensidade com que partículas são capturadas" .

Intuitivamente sabemos que os valores $p_{x}$ e $q_{x}$ são, conjuntamente, responsáveis pela média do número de partículas que se deslocam para a direita ou para a esquerda, respectivamente, caracterizando a deriva no meio aleatório. Assim, influem fundamentalmente para a classificação de um processo.

Mas, como perceber esta e todas as influências necessárias através das distribuições $Q_{i}$ que são responsáveis pelo meio aleatório gerado? Como isto pode ser percebido através das funções $h$ e $H$ ?

Os teoremas 3.1 .1 e 3.1 .2 a seguir constituem o resultado principal deste estudo que é uma forma de classificar o processo. 


\subsection{Principais Resultados}

Teorema 3.1.1 Para que $\mathbf{E} \nu$ seja finita (P-a.s.), é necessário e suficiente que existam $\lambda_{1}, \ldots, \lambda_{k}>0$ tais que

$$
\lambda_{i+1} \leq H_{i}\left(\lambda_{i}\right)
$$

para $i=1, \ldots, k-1, e$

$$
\lambda_{1} \leq H_{k}\left(\lambda_{k}\right)
$$

Além disso,

(i) se $\prod_{i=1}^{k} \lambda_{i}<1$ então o processo é fortemente transiente $\mathbf{P}$-q.c.;

(ii) se $\prod_{i=1}^{k} \lambda_{i}>1$ então o processo é fortemente recorrente $\mathbf{P}$-q.c.

Teorema 3.1.2 Não havendo solução para as equações 3.1 e 3.2, o processo é classificado como recorrente ( não fortemente) P-a.s.

Lema 3.1.1 Quaisquer que sejam os valores $\lambda_{1}, \ldots, \lambda_{k}>0$ satisfazendo 3.1 e 3.2, não se verifica $\prod_{i=1}^{k} \lambda_{i}=1$

A demonstração do teorema 3.1.2 constitui uma adaptação direta do teorema 4.3 em Comets et al.[11].

Segundo os teoremas acima, temos, por exclusão, que o processo não pode ser classificado como transiente - não fortemente. 


\subsection{Um Lema Importante}

Antes de expormos a demonstração dos resultados recém enunciados, faremos uso dos teoremas 2.1 e 2.2 apresentados em Comets et al.[11] e aqui enunciados pelo seguinte lema.

Lema 3.2.1 Para Ev ser finita é necessário que exista uma função positiva $f(x)$ tal que para todo $x \neq 0$

$$
p(x) f(x-1)+s(x) f(x)+q(x) f(x+1)=\frac{f(x)}{r(x)}
$$

É suficiente para Eע ser finita que exista uma função positiva $f(x)$ tal que para todo $x \neq 0$

$$
p(x) f(x-1)+s(x) f(x)+q(x) f(x+1) \leq \frac{f(x)}{r(x)}
$$

Além disso:

Se $f(x) \rightarrow 0 \operatorname{com} x \rightarrow \infty$, então o processo é fortemente transiente.

Se $f(x) \rightarrow \infty \operatorname{com} x \rightarrow \infty$, então o processo é fortemente recorrente.

\section{Prova do Lema 3.1.1}

Sabemos que se $r^{\xi} \geq 1, \forall \xi \in M$, então:

$$
\frac{1}{r^{\xi}} \leq 1 \Rightarrow \frac{1}{r^{\xi}} \leq p^{\xi}+s^{\xi}+q^{\xi} \quad \Rightarrow \quad \frac{1}{q^{\xi}}\left(\frac{1}{r^{\xi}}-s^{\xi}-p^{\xi}\right) \leq 1 \quad \forall \xi \in M,
$$

isto é

$$
h^{\xi}(1) \leq 1 \quad \forall \xi \in M
$$

o que torna

$$
H_{i}(1) \leq 1 \quad \forall i \in\{1,2, \ldots, k\}
$$

Além disso, para algum $j_{0} \in\{1,2, \ldots, k\}$ temos

$$
Q_{j_{0}}\left\{\xi \in M: r^{\xi}>1\right\}>0
$$


e assim existe $\xi^{*} \in \operatorname{supp} Q_{j_{0}}$ tal que $r^{\xi^{*}}>1$, o que torna, segundo procedimento análogo,

$$
h^{\xi^{*}}(1)<1
$$

e portanto

$$
H_{j_{0}}(1)<1
$$

Tendo isto em mente, suponha que existam $\lambda_{1}, \lambda_{2}, \ldots, \lambda_{k}$ satisfazendo as condições do teorema 3.1 e com $\prod_{i=1}^{k} \lambda_{i}=1$. Assim, teríamos obrigatoriamente só dois casos possíveis:

$\mathbf{1}^{\mathrm{o}}$ caso: $\lambda_{1}=\lambda_{2}=\cdots=\lambda_{k}=1$.

Sendo $\lambda_{k+1}=\lambda_{1}$ e $j_{0} \in\{1,2, \ldots, k\}$ tal que $Q_{j_{0}}\left\{\xi \in M: r^{\xi}>1\right\}>0$, vemos não ser possível $\lambda_{j_{0}+1}=1$ pois

$$
\lambda_{j_{0}+1} \leq H_{j_{0}}\left(\lambda_{j_{0}}\right)<1
$$

$\mathbf{2}^{\mathbf{o}}$ caso: Existiriam $i, j \in\{1,2 \ldots, k\}$ tais que $\lambda_{i}<1$ e $\lambda_{j}>1$ com $i \neq j$.

Se $\lambda_{i}<1$, por 2.3 e 3.5 , sabemos que

$$
h^{\xi}\left(\lambda_{i}\right) \leq h^{\xi}(1) \quad \Rightarrow \quad H_{i}\left(\lambda_{i}\right) \leq H_{i}(1) \leq 1 \quad \forall \xi \in M,
$$

e sendo

$$
\lambda_{i+1} \leq H_{i}\left(\lambda_{i}\right) \leq 1
$$

temos

$$
\lambda_{i}<1 \quad \Rightarrow \quad \lambda_{i+1} \leq 1
$$

Segundo procedimento análogo, teríamos que

$$
\lambda_{i+1} \leq 1 \Rightarrow \lambda_{i+2} \leq 1 \Rightarrow \cdots \Rightarrow \lambda_{k} \leq 1 \Rightarrow \lambda_{1} \leq 1 \cdots \Rightarrow \lambda_{i-1} \leq 1 .
$$

Isto é, uma contradição, pois não haveria $\lambda_{j}>1$ como suposto. 


\subsection{A Condição Suficiente}

Vejamos porque a existência de solução para 3.1 e 3.2 é condição suficiente para que tenhamos $\mathrm{E} \nu<\infty$.

Nossa estratégia será mostrar que uma solução qualquer de 3.1 e 3.2 implica na existência de uma função $f$ satisfazendo 3.3 e portanto, como afirma o lema 3.2.1, $\mathrm{E} \nu$ será finita.

Suponhamos que exista tal solução e seja $x=n k+j$ um sítio não nulo de $\mathbb{Z}_{+}$ onde $n \in \mathbb{Z}_{+}$e $1 \leq j \leq k$.

Sendo $\lambda_{j+1} \leq H_{j}\left(\lambda_{j}\right)$, sabemos que para todo $\xi \in \operatorname{supp} Q$

$$
\lambda_{j+1} \leqslant \frac{1}{q^{\xi}(x)}\left(\frac{1}{r^{\xi}(x)}-s^{\xi}(x)-\frac{p^{\xi}(x)}{\lambda_{j}}\right), \quad \text { onde } \quad \lambda_{j+1}=\lambda_{1} \quad \text { se } \quad j=k,
$$

e portanto

$$
p^{\xi}(x)+s^{\xi}(x) \lambda_{j}+q^{\xi}(x) \lambda_{j} \lambda_{j+1} \leqslant \frac{\lambda_{j}}{r^{\xi}(x)} .
$$

Multiplicando ambos os membros da inequação acima pelo termo positivo

$$
\left(\prod_{i=1}^{k} \lambda_{i}\right)^{n} \prod_{i=1}^{j-1} \lambda_{i} \quad \text { onde } \quad \prod_{i=1}^{j-1} \lambda_{i}=1 \quad \text { se } \quad j=1
$$

temos

$$
\begin{aligned}
p^{\xi}(x)\left(\prod_{i=1}^{k} \lambda_{i}\right)^{n} \prod_{i=1}^{j-1} \lambda_{i} & +s^{\xi}(x)\left(\prod_{i=1}^{k} \lambda_{i}\right)^{n}\left(\prod_{i=1}^{j-1} \lambda_{i}\right) \lambda_{j}+ \\
& +q^{\xi}(x)\left(\prod_{i=1}^{k} \lambda_{i}\right)^{n}\left(\prod_{i=1}^{j-1} \lambda_{i}\right) \lambda_{j} \lambda_{j+1} \leqslant \frac{\left(\prod_{i=1}^{k} \lambda_{i}\right)^{n}\left(\prod_{i=1}^{j-1} \lambda_{i}\right) \lambda_{j}}{r^{\xi}(x)}
\end{aligned}
$$

ou seja,

$$
\begin{aligned}
p^{\xi}(x)\left(\prod_{i=1}^{k} \lambda_{i}\right)^{n} \prod_{i=1}^{j-1} \lambda_{i} & +s^{\xi}(x)\left(\prod_{i=1}^{k} \lambda_{i}\right)^{n} \prod_{i=1}^{j} \lambda_{i}+ \\
& +q^{\xi}(x)\left(\prod_{i=1}^{k} \lambda_{i}\right)^{n} \prod_{i=1}^{j+1} \lambda_{i} \leqslant \frac{\left(\prod_{i=1}^{k} \lambda_{i}\right)^{n} \prod_{i=1}^{j} \lambda_{i}}{r^{\xi}(x)} \quad \forall x \in \mathbb{Z}_{+} .
\end{aligned}
$$


Isto nos conduz a identificar a seguinte função positiva $f(x)=\left\{\begin{array}{c}1 \quad \text { se } x=0 \\ \left(\prod_{i=1}^{k} \lambda_{i}\right)^{n} \prod_{i=1}^{j} \lambda_{i} \text { se } x \neq 0 \quad \text { onde } x=n k+j \quad \text { com } n \in \mathbb{Z}_{+} \quad \text { e } 1 \leqslant j \leqslant k\end{array}\right.$

que satisfaz 3.3 para todo $\xi \in \operatorname{supp} Q$, pois vemos em 3.6 que

$$
p^{\xi}(x) f(x-1)+s^{\xi}(x) f(x)+q^{\xi}(x) f(x+1) \leq \frac{f(x)}{r^{\xi}(x)} .
$$

Assim, dados $\lambda_{1}, \ldots, \lambda_{k}>0$ solução de 3.3 e 3.4, a função acima, construída com essa solução, satisfaz 4.2 o que garante ser finita $\mathrm{E} \nu$.

Além disso,

$$
\begin{array}{ll}
\text { se } \prod_{i=1}^{k} \lambda_{i}<1 & \text { o processo é fortemente transiente; } \\
\text { se } \quad \prod_{i=1}^{k} \lambda_{i}>1 \quad \text { o processo é fortemente recorrente. }
\end{array}
$$

\section{Prova}

Supondo que haja uma solução para 3.1 e 3.2 e sabendo que não se verifica $\prod_{i=1}^{k} \lambda_{i}=1$, mostremos que:

$$
\begin{array}{ll}
\text { se } & \prod_{i=1}^{k} \lambda_{i}<1 \Rightarrow f(x) \stackrel{n \rightarrow \infty}{\longrightarrow} 0 \\
\text { se } & \prod_{i=1}^{k} \lambda_{i}>1 \Rightarrow f(x) \stackrel{n \rightarrow \infty}{\longrightarrow} \infty
\end{array}
$$

Sejam $\mathcal{M}$ e $\boldsymbol{m}$, respectivamente, o elemento máximo e o elemento mínimo do conjunto

$$
\left\{\lambda_{1}, \prod_{i=1}^{2} \lambda_{i}, \ldots, \prod_{i=1}^{k} \lambda_{i}\right\}
$$

construído, assim como a função $f$, a partir desta solução.

Para $x>0$, sabemos que

$$
0<\left(\prod_{i=1}^{k} \lambda_{i}\right)^{n} \boldsymbol{m} \leq f(x) \leq\left(\prod_{i=1}^{k} \lambda_{i}\right)^{n} \mathcal{M}
$$


Onde $x=n k+j$ com $n \in \mathbb{Z}_{+}$e $1 \leq j \leq k$.

Se $x \rightarrow \infty$ então $n \rightarrow \infty$ e portanto

$$
\begin{aligned}
& \text { se } \prod_{i=1}^{k} \lambda_{i}<1 \Rightarrow\left(\prod_{i=1}^{k} \lambda_{i}\right)^{n} \mathcal{M} \stackrel{x \rightarrow \infty}{\longrightarrow} 0 \Rightarrow f(x) \stackrel{x \rightarrow \infty}{\longrightarrow} 0 ; \\
& \text { se } \prod_{i=1}^{k} \lambda_{i}>1 \Rightarrow\left(\prod_{i=1}^{k} \lambda_{i}\right)^{n} \boldsymbol{m} \stackrel{x \rightarrow \infty}{\longrightarrow} \infty \Rightarrow f(x) \stackrel{x \rightarrow \infty}{\longrightarrow} \infty .
\end{aligned}
$$

\subsection{A Condição Necessária}

Demonstraremos que a existência de solução para 3.1 e 3.2 é condição necessária para que $E \nu$ seja finita, como foi enunciado no teorema 3.1.1.

Para isso, suponha por absurdo que $E \nu<\infty$ mas que não existam $\lambda_{1}, \ldots, \lambda_{k}>0$ satisfazendo 3.1 e 3.2 .

Pelo lema 3.2.1, sendo $E \nu<\infty$, existirá uma função positiva $f$ que satisfaz 3.3. Esta função pode ser determinada pela seqüência de quocientes

$$
\rho_{x+1}=\frac{f(x+1)}{f(x)}, \quad x=0,1,2, \ldots
$$

juntamente, é claro, com o valor de $f(0)$.

Assim, para os sítios $x=1,2,3, \ldots$ temos

$$
\begin{array}{ll}
p(x) f(x-1)+s(x) f(x)+q(x) f(x+1)=\frac{f(x)}{r(x)} & \Longleftrightarrow \\
\frac{p(x) f(x-1)}{f(x)}+\frac{s(x) f(x)}{f(x)}+\frac{q(x) f(x+1)}{f(x)}=\frac{f(x)}{r(x) f(x)} & \Longleftrightarrow \\
\frac{q(x) f(x+1)}{f(x)}=\frac{1}{r(x)}-s(x)-\frac{p(x) f(x-1)}{f(x)} & \Longleftrightarrow \\
\frac{f(x+1)}{f(x)}=\frac{1}{q(x)}\left(\frac{1}{r(x)}-s(x)-\frac{p(x)}{f(x) / f(x-1)}\right) &
\end{array}
$$


Lembrando que para o sítio $x$, segundo o meio $\boldsymbol{\xi}$ fixado, temos

$$
p(x)=p^{\xi_{x}}, \quad s(x)=s^{\xi_{x}}, \quad q(x)=q^{\xi_{x}} \quad \text { e } \quad r(x)=r^{\xi_{x}}
$$

resulta que

$$
\frac{f(x+1)}{f(x)}=\frac{1}{q^{\xi_{x}}}\left(\frac{1}{r^{\xi_{x}}}-s^{\xi_{x}}-\frac{p^{\xi_{x}}}{f(x) / f(x-1)}\right)
$$

isto é,

$$
\begin{gathered}
\rho_{x+1}=\frac{1}{q^{\xi_{x}}}\left(\frac{1}{r^{\xi_{x}}}-s^{\xi_{x}}-\frac{p^{\xi_{x}}}{\rho_{x}}\right) \\
\rho_{x+1}=h^{\xi_{x}}\left(\rho_{x}\right), \quad x=1,2,3, \ldots
\end{gathered}
$$

Por esta relação recorrente, a seqüência positiva $\left\{\rho_{x}\right\}_{x \in \mathbb{N}}$ pode ser determinada por $f(0)$ e o meio $\boldsymbol{\xi}$.

Nossa meta é mostrar que caso não haja solução para 3.1 e 3.2, não existe uma seqüência positiva $\left\{\rho_{x}\right\}_{x \in \mathbb{N}}$ como em 3.7. Isto, por sua vez, implica na inexistência de uma função positiva $f$ satisfazendo 3.3 e, pelo Lema 3.2.1, $E \nu=\infty$, produzindo uma contradição.

Assim, podemos afirmar que, se $E \nu<\infty$, então teremos necessariamente uma solução para 3.1 e 3.2 .

Para tanto, precisamos de algumas definições e teoremas.

Definição 3.4.1 Chamaremos de $\left\{\beta_{j}^{i}\right\}, i=1, \ldots, k, j \geq 1$ a seqüência

$$
\beta_{1}^{1}, \beta_{1}^{2}, \ldots, \beta_{1}^{k}, \beta_{2}^{1}, \beta_{2}^{2}, \ldots, \beta_{2}^{k}, \beta_{3}^{1}, \beta_{3}^{2}, \ldots, \beta_{3}^{k}, \ldots
$$

onde

$$
\begin{aligned}
& \beta_{j}^{i+1}=H_{i}\left(\beta_{j}^{i}\right), \quad i=1,2, \ldots, k-1 \\
& \beta_{j+1}^{1}=H_{k}\left(\beta_{j}^{k}\right)
\end{aligned}
$$

Teorema 3.4.1 Se para uma escolha de $0<\beta_{1}^{1} \leq \infty$ a seqüência $\left\{\beta_{j}^{i}\right\}$ possuir todos os termos positivos então há solução para 3.1 e 3.2. 


\section{Prova.}

Suponha os termos da seqüência $\left\{\beta_{j}^{i}\right\}$ positivos.

A sub-seqüência $\left\{\beta_{j}^{1}\right\}_{j \geq 1}$ é tal que

$$
\beta_{j+1}^{1}=\mathcal{H}\left(\beta_{j}^{1}\right)
$$

pela definição da seqüência $\left\{\beta_{j}^{i}\right\}$.

Naturalmente, a sub-seqüência $\left\{\beta_{j}^{1}\right\}_{j \geq 1}$ pode se comportar como estritamente decrescente ou não.

Se a sub-seqüência positiva $\left\{\beta_{j}^{1}\right\}_{j \geq 1}$ for estritamente decrescente, então existe

$$
\lim _{j \rightarrow \infty} \beta_{j}^{1}=\beta^{*}
$$

Como $\mathcal{D}=\left[\lambda_{0} ; \infty\right)$ para algum $\lambda_{0}>0$ então $\mathcal{D}$ é fechado e portanto $\beta^{*} \in \mathcal{D}$.

Segundo as características de $\mathcal{H}$ citadas no corolário 2.2.1, temos que

$$
\beta^{*}=\mathcal{H}\left(\beta^{*}\right)
$$

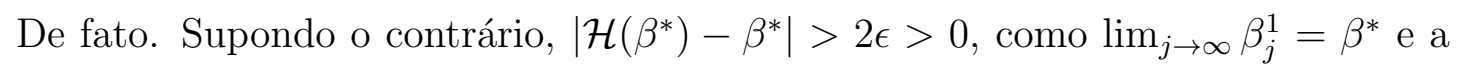
função $\mathcal{H}$ é contínua, existe $\boldsymbol{j}$ tal que

$$
\left|\mathcal{H}\left(\beta^{*}\right)-\mathcal{H}\left(\beta_{j}^{1}\right)\right|<\epsilon \quad \text { e } \quad \beta_{j}^{1}-\beta^{*}<\epsilon .
$$

Notando que $\mathcal{H}\left(\beta_{j}^{1}\right)=\beta_{j+1}^{1}$, temos

$$
\left|\mathcal{H}\left(\beta^{*}\right)-\beta^{*}\right|>2 \epsilon>0, \quad\left|\mathcal{H}\left(\beta^{*}\right)-\beta_{j+1}^{1}\right|<\epsilon \quad \text { e } \quad \beta_{j}^{1}-\beta^{*}<\epsilon
$$

e assim,

$$
\left|\mathcal{H}\left(\beta^{*}\right)-\beta^{*}\right|-\left|\mathcal{H}\left(\beta^{*}\right)-\beta_{j+1}^{1}\right|>2 \epsilon-\epsilon=\epsilon
$$

mas

$$
\left|\left(\mathcal{H}\left(\beta^{*}\right)-\beta^{*}\right)-\left(\mathcal{H}\left(\beta^{*}\right)-\beta_{\boldsymbol{j}+1}^{1}\right)\right| \geq\left|\mathcal{H}\left(\beta^{*}\right)-\beta^{*}\right|-\left|\mathcal{H}\left(\beta^{*}\right)-\beta_{\boldsymbol{j}+1}^{1}\right|>\epsilon .
$$

e portanto

$$
\begin{aligned}
& \left|\mathcal{H}\left(\beta^{*}\right)-\beta^{*}-\mathcal{H}\left(\beta^{*}\right)+\beta_{j+1}^{1}\right|>\epsilon \\
& \left|\beta_{j+1}^{1}-\beta^{*}\right|>\epsilon
\end{aligned}
$$


Tendo-se

$$
\left|\beta_{\boldsymbol{j}+1}^{1}-\beta^{*}\right|>\epsilon \quad \text { e } \quad \beta_{\boldsymbol{j}}^{1}-\beta^{*}<\epsilon \quad \Longrightarrow \quad \beta_{\boldsymbol{j}+1}^{1}<\beta^{*} \quad \text { ou } \quad \beta_{\boldsymbol{j}}^{1}<\beta_{\boldsymbol{j}+1}^{1},
$$

em ambos os casos uma contradição, pois supomos $\left\{\beta_{j}^{1}\right\}$ positiva e estritamente decrescente.

Então, sendo $\beta^{*}=\mathcal{H}\left(\beta^{*}\right)$, o conjunto de valores

$$
\begin{aligned}
& \lambda_{1}=\beta^{*} \\
& \lambda_{i}=H_{i-1} \circ \cdots \circ H_{1}\left(\beta^{*}\right)
\end{aligned}
$$

satisfaz as equações 3.1 e 3.2 pois, por construção, temos não só $\lambda_{i} \leq H_{i-1}\left(\lambda_{i-1}\right)$ para $i=2, \ldots, k$, mas $\lambda_{i}=H_{i-1}\left(\lambda_{i-1}\right)$. Como $H_{k}\left(\lambda_{k}\right)=H_{k} \circ \cdots \circ H_{1}\left(\beta^{*}\right)=\mathcal{H}\left(\beta^{*}\right)=\beta^{*}$, também temos $\lambda_{1}=\mathcal{H}\left(\lambda_{2}\right)$.

Não sendo $\left\{\beta_{j}^{1}\right\}$ estritamente decrescente teremos

$$
\beta_{\boldsymbol{j}}^{1} \leqslant \beta_{\boldsymbol{j}+1}^{1} \text { para algum } \boldsymbol{j} \geq 1
$$

Neste caso temos como possível solução

$$
\lambda_{i}=\beta_{j}^{i}, \quad i=1,2, \ldots, k
$$

De fato. Por construção e pela definição de $\left\{\beta_{j}^{1}\right\}$, temos

$$
\lambda_{i}=\beta_{j}^{i}=H_{i-1}\left(\beta_{j}^{i-1}\right)
$$

e como $\lambda_{i-1}=\beta_{j}^{i-1}$,

$$
\lambda_{i}=H_{i-1}\left(\lambda_{i-1}\right) \text { para } i=2, \ldots, k
$$

Sendo $\lambda_{1}=\beta_{j}^{1} \leqslant \beta_{j+1}^{1}$ e $\beta_{j+1}^{1}=H_{k}\left(\beta_{j}^{k}\right)=H_{k}\left(\lambda_{k}\right)$, temos

$$
\lambda_{1} \leq H_{k}\left(\lambda_{k}\right)
$$

Corolário 3.4.1 Não havendo uma solução para 3.1 e 3.2, qualquer que seja a escolha de $0<\beta_{1}^{1} \leq \infty$ haverá um elemento negativo na seqüência

$$
\beta_{1}^{1}, \beta_{1}^{2}, \ldots, \beta_{1}^{k}, \beta_{2}^{1}, \beta_{2}^{2}, \ldots, \beta_{2}^{k}, \beta_{3}^{1}, \beta_{3}^{2}, \ldots, \beta_{3}^{k}, \ldots
$$

Prova. : Imediata. 
Teorema 3.4.2 Se na seqüência $\left\{\beta_{j}^{i}\right\}$, onde $\beta_{1}^{1}=\infty$, há um termo negativo então não existe uma seqüência positiva $\left\{\rho_{x}\right\}_{x \in \mathbb{N}}$ como em 3.7. qualquer que seja o meio $\boldsymbol{\xi}$.

\section{Prova.}

Como sabemos, a seqüência $\left\{\beta_{j}^{i}\right\}$ é construída recursivamente a partir de $\beta_{1}^{1}=\infty$ por aplicações das funções $H_{i}$. Chamaremos de $\left\{\bar{\beta}_{j}^{i}\right\}, i=1,2,3, \ldots, k$ e $j \geq 1$, uma seqüência resultante de $\left\{\beta_{j}^{i}\right\}$ onde

$$
\begin{aligned}
& \bar{\beta}_{1}^{1}=\beta_{1}^{1}=\infty \\
& \bar{\beta}_{j}^{i+1}=h^{\xi_{x}}\left(\bar{\beta}_{j}^{i}\right), \quad i=1,2, \ldots, k-1 \\
& \bar{\beta}_{j+1}^{1}=h^{\xi_{x}}\left(\bar{\beta}_{j}^{k}\right),
\end{aligned}
$$

sendo $x$ o sítio $k(j-1)+i, i=1,2, \ldots, k$ e $j=1,2, \ldots$

Como se vê, a seqüência $\left\{\bar{\beta}_{j}^{i}\right\}$ depende de um meio $\boldsymbol{\xi}=\left\{\xi_{1}, \xi_{2}, \ldots\right\}$. Entretanto, qualquer que seja o meio $\boldsymbol{\xi}$, temos

$$
h^{\xi_{1}}\left(\bar{\beta}_{1}^{1}\right) \geq H_{1}\left(\beta_{1}^{1}\right) \text {, ou seja }, \bar{\beta}_{1}^{2} \geq \beta_{1}^{2} .
$$

Isto se dá pela própria definição de $H_{i}$, para todo $i$, e porque se tem $\bar{\beta}_{1}^{1}=\beta_{1}^{1}$. Conseqüentemente, sendo $\bar{\beta}_{1}^{2} \geq \beta_{1}^{2}$, temos

$$
h^{\xi_{2}}\left(\bar{\beta}_{1}^{2}\right) \geq H_{2}\left(\beta_{1}^{2}\right) \quad \text {, ou seja }, \quad \bar{\beta}_{1}^{3} \geq \beta_{1}^{3} .
$$

Por indução, é fácil ver que, qualquer que seja o meio $\boldsymbol{\xi}$, a seqüência $\left\{\bar{\beta}_{j}^{i}\right\}$ majora tanto $\left\{\beta_{j}^{i}\right\}$ como toda seqüência $\left\{\alpha_{j}^{i}\right\}$, também dependente do mesmo meio $\boldsymbol{\xi}$, onde

$$
\begin{aligned}
& 0<\alpha_{1}^{1} \leq \infty, \\
& \alpha_{j}^{i+1}=h^{\xi_{x}}\left(\alpha_{j}^{i}\right), \quad i=1,2, \ldots, k-1 \\
& \alpha_{j+1}^{1}=h^{\xi_{x}}\left(\alpha_{j}^{k}\right),
\end{aligned}
$$

sendo $x$ o sítio $k(j-1)+i, i=1,2, \ldots, k$ e $j=1,2, \ldots$

Pois sendo $\alpha_{1}^{1} \leq \bar{\beta}_{1}^{1}$ e pela monotonicidade da função $h$, qualquer que seja o meio $\boldsymbol{\xi}$, temos

$$
h^{\xi_{1}}\left(\alpha_{1}^{1}\right) \leq h^{\xi_{1}}\left(\bar{\beta}_{1}^{1}\right), \text { ou seja }, \quad \alpha_{1}^{2} \leq \bar{\beta}_{1}^{2},
$$

e portanto

$$
\alpha_{1}^{n} \leq \bar{\beta}_{1}^{n}, \quad \forall n \geq 1
$$


Com isto em mente, suponha ser $N=k \boldsymbol{j}+\boldsymbol{i}$ a posição do primeiro termo negativo na seqüência $\left\{\beta_{j}^{i}\right\}$, com $\beta_{1}^{1}=\infty$.

Fixado um $\epsilon>0$, defina

$$
A_{N}^{(\epsilon)}:=\left\{\left(\xi_{1}, \xi_{2}, \ldots, \xi_{N-1}\right) \in M^{N-1}: h^{\xi_{n}}\left(\bar{\beta}_{j}^{i}\right) \leq H_{i}\left(\beta_{j}^{i}\right)+\epsilon\right\}
$$

sendo $0<n<N$, com $n=k(j-1)+i$ onde $j \geq 1$ e $1 \leq i \leq k$,

Suponha um meio $\boldsymbol{\xi}=\left(\xi_{1}, \xi_{2}, \ldots\right)$ onde $\left(\xi_{1}, \xi_{2}, \ldots, \xi_{N-1}\right) \in A_{N}^{(\epsilon)}$ de forma que $0<\epsilon<\left|\beta_{j}^{i}\right|$, isto é, $\epsilon>0$ e $\beta_{j}^{i}+\epsilon<0$. Neste caso, temos $\bar{\beta}_{j}^{i}<0$ pois

$$
\bar{\beta}_{j}^{i}=h^{\xi_{N-1}}\left(\beta_{j}^{i-1}\right) \leq H_{i-1}\left(\beta_{j}^{i-1}\right)+\epsilon=\beta_{j}^{i}+\epsilon<0
$$

onde

$$
\beta_{j}^{i-1}=\left\{\begin{array}{ccc}
\beta_{j}^{i-1} & \text { se } & 1<i \leqslant k \\
\beta_{j-1}^{k} & \text { se } & i=1
\end{array}\right.
$$

Conseqüentemente, sabendo que $\left\{\bar{\beta}_{j}^{i}\right\}$ majora $\left\{\alpha_{j}^{i}\right\}$ qualquer que seja $0<\alpha_{1}^{1} \leq \infty$, também temos $\alpha_{j}^{i}<0$.

Assim, para um processo desenvolvendo-se em tal meio, teríamos $\rho_{N}<0$ para quaisquer valores de $f(0)$ e $f(1)$, pois a seqüência $\left\{\rho_{x}\right\}_{x \in \mathbb{N}}$ é idêntica à seqüência $\left\{\alpha_{j}^{i}\right\} \operatorname{com} \alpha_{1}^{1}=\frac{f(1)}{f(0)}$.

Evidentemente, não podemos garantir que exatamente $\rho_{N}$ será negativo. Certamente isto ocorre se o meio $\boldsymbol{\xi}$ dispor elementos $\xi_{x}$ nos sítio de $\mathbb{Z}_{+}$de tal forma que $\left(\xi_{1}, \xi_{2}, \ldots, \xi_{N-1}\right) \in A_{N}^{(\epsilon)}$ para um $\epsilon$ suficientemente pequeno.

No entanto, para evidenciar a existência de um termo negativo em qualquer seqüência $\left\{\rho_{x}\right\}_{x \in \mathbb{N}}$ com $0<\rho_{1} \leq+\infty$ é suficiente mostrar que, qualquer que seja o meio $\boldsymbol{\xi}$ onde se desenvolva o processo, existe um inteiro $0 \leq \tau<\infty$ e $\tau / k \in \mathbb{Z}_{+}$de forma que $\left(\xi_{\tau+1}, \xi_{\tau+2}, \ldots, \xi_{\tau+(N-1)}\right) \in A_{\tau+N}^{(\epsilon)}$. Assim, para quaisquer valores de $f(0)$ e $f(1)$, teríamos $\rho_{k \tau+1}<\infty$ e portanto $\rho_{k \tau+N}<0$. Para este fim, vejamos:

Para qualquer $\epsilon>0$ e qualquer sítio $n=k(j-1)+i$, com $0<n<N$, seja $A_{n}^{\epsilon}:=\left\{\xi \in M: h^{\xi}\left(\beta_{j}^{i}\right) \leq H_{i}\left(\beta_{j}^{i}\right)+\epsilon\right\}$. Particularmente para $n=1$, se $\xi_{1} \in A_{1}^{\epsilon}$ então não só

$$
h^{\xi_{1}}\left(\beta_{1}^{1}\right) \leq H_{1}\left(\beta_{1}^{1}\right)+\epsilon
$$

mas

$$
h^{\xi_{1}}\left(\bar{\beta}_{1}^{1}\right) \leq H_{1}\left(\beta_{1}^{1}\right)+\epsilon,
$$


pois $\beta_{1}^{1}=\bar{\beta}_{1}^{1}$.

Segundo a definição de $H_{i}$, para quaisquer $\epsilon>0$ e $\lambda>0$, temos que

$$
\mathbf{P}\left\{\xi \in M: h^{\xi}(\lambda) \leq H_{i}(\lambda)+\epsilon\right\}>0,
$$

Para todo $n=k(j-1)+i$, com $0<n<N, j \geq 1$ e $1 \leq i \leq k$, fazendo $\lambda=\beta_{j}^{i}>0$, qualquer que seja $\epsilon>0$ temos

$$
\mathbf{P}\left\{\xi_{i} \in M: h^{\xi_{i}}\left(\beta_{j}^{i}\right) \leq H_{i}\left(\beta_{j}^{i}\right)+\epsilon\right\}>0
$$

isto é,

$$
\mathbf{P}\left\{\xi_{i} \in A_{n}^{\epsilon}\right\}>0
$$

e pela propriedade P3 temos que

$$
\mathbf{P}\left\{\xi_{1} \in A_{1}^{\epsilon}, \ldots, \xi_{N-1} \in A_{N-1}^{\epsilon}\right\}>0 .
$$

Assim, para $\epsilon$ suficientemente pequeno, temos

$$
\mathbf{P}\left\{A_{N}^{(\epsilon)}\right\}>0 .
$$

Como temos $\mathbf{P}\left\{A_{N}^{(\epsilon)}\right\}>0$ para $\epsilon$ suficientemente pequeno, a propriedade P2, que possui qualquer meio $\boldsymbol{\xi}$, garante a existência de $\tau$ como desejado. Isto é,

$$
\left(\xi_{\tau+1}, \xi_{\tau+2}, \ldots, \xi_{\tau+(N-1)}\right) \in A_{\tau+N}^{(\epsilon)}
$$

para algum inteiro $0 \leq \tau<\infty$ qualquer que seja o meio $\boldsymbol{\xi}$ onde se desenvolverá o processo.

Desta forma, fica provado que, se na seqüência $\left\{\beta_{j}^{i}\right\}$, onde $\beta_{1}^{1}=\infty$, há um termo negativo então não existe uma seqüência positiva $\left\{\rho_{x}\right\}_{x \in \mathbb{N}}$ como em 3.7 qualquer que seja o meio $\boldsymbol{\xi}$.

Assim, se $E \nu<\infty$ teremos necessariamente uma solução para 3.1 e 3.2 , pois caso contrário, como visto no Corolário 3.4.1, haveria um termo negativo na seqüência $\left\{\beta_{j}^{i}\right\}$ qualquer que fosse o valor de $\beta_{1}^{1}$ ( em particular para $\beta_{1}^{1}=\infty$ ) e, pelo Teorema 3.4.2, não haveria uma seqüência positiva $\left\{\rho_{x}\right\}_{x \in \mathbb{N}}$ como em 3.7. A inexistência de tal seqüência implica em não haver uma função positiva $f$ satisfazendo 3.3 no lema 3.2.1 e assim sendo, por este mesmo lema, deveríamos ter $E \nu=\infty$ : uma contradição. 


\section{Referências Bibliográficas}

[1] J. B. Baillon, Ph. Clément, A. Greven, and F. den Hollander. A variational approach to branching random walk in random environment. Ann. Probab., 21(1):290-317, 1993.

[2] F. den Hollander, M. V. Menshikov, and S. Yu. Popov. A note on transience vs. recurrence for branching random walk in a random environment. J. Statist. Phys., 95(3/4):587-614, 1999.

[3] K. Fleischmann and A. Greven. Localization and selection in a mean field branching random walk in a random environment. Ann. Probab., 20(4):2141-2163, 1992.

[4] M. Menshikov G. Fayolle, V. Malyshev. Topics in the contructive theory of countable Markov chains. Cambridge University Press, 1995.

[5] A. Greven and F. den Hollander. Branching random walk in random in a random environment; phase transition for local and global growth rates. Probab. Theory Relat., Fields 91(2):195-249, 1992.

[6] F. I. Karpelevich, M. Ya. Kelbert, and Yu. M. Suhov. The boundedness of branching Markov processes. In M. Freidlin, editor, The Dynkin Festschrift. Markov Processes and their Applications, number 34 in Progress in probability, pages 143-153. Birkhauser, Boston, 1994.

[7] F. I. Karpelevich and Yu. M. Suhov. A criterion of bouddedness of discrete branching random walk. In K. Atreya and P. Jagers, editors, Classical and 
Modern Branching Processes, number 84 in IMA volumes and mathematics and its applications, pages 141-156. Springer-Verlag, New York, 1996.

[8] F. I. Karpelevich and Yu. M. Suhov. Boundedness of one-dimensional branching markov processes. J. Appl. Math. Stochastic Anal, 10(4):307-332, 1997.

[9] F. Machado and S. Yu. Popov. Branching random walk in a markovian random environment. Journal of Applied Probability, 37(4):1157 - 1163, 2001.

[10] F. Comets M. V. Menshikov and S. Yu. Popov. Lyapunov functions for random walks and strings in random environment. Ann. Probab., 26(4):1433-1445, 1998.

[11] F. Comets M. V. Menshikov and S. Yu. Popov. One-dimensional branching random walk in random environment: a classification. Markov Process Relat., Fields 4(4):465-477, 1998.

[12] M. V. Menshikov and S. E. Volkov. Branching markov chains: qualitative characteristics. Markov Processes Relat., Fields 3(2):225-241, 1997.

[13] P. Révész. Supercritical branching random walk in d-dimensional random environment. Applied Statistical Science, III:41-51, 1998. 